\title{
Evolution of the star formation efficiency in galaxies
}

\section{Jonathan Braine}

Laboratoire d'Astrophysique de Bordeaux France, email: braine@obs.u-bordeaux1.fr

\begin{abstract}
The physical and chemical evolution of galaxies is intimately linked to star formation, We present evidence that molecular gas $\left(\mathrm{H}_{2}\right)$ is transformed into stars more quickly in smaller and/or subsolar metallicity galaxies than in large spirals - which we consider to be equivalent to a star formation efficiency (SFE). In particular, we show that this is not due to uncertainties in the $\mathrm{N}\left(\mathrm{H}_{2}\right) / \mathrm{I}_{c o}$ conversion factor. Several possible reasons for the high SFE in galaxies like the nearby M33 or NGC 6822 are proposed which, separately or together, are the likely cause of the high SFE in this environment. We then try to estimate how much this could contribute to the increase in cosmic star formation rate density from $z=0$ to $z=1$.
\end{abstract}

Keywords. Stars: Formation, Interstellar Medium, Galaxies: M33, Galaxies: Local Group

\section{References}

Braine J., Gratier P., Kramer C., Schuster K. F., Tabatabaei F., \& Gardan E., 2010, A\&A, 520, 107

Braine J., et al., 2010, A\& $A$, 518, L69

Combes F., García-Burillo S., Braine J., Schinnerer E., Walter F., \& Colina L., 2013, A\&\&A, 550, 41

Combes F., García-Burillo S., Braine J., Schinnerer E., Walter F., \& Colina L., 2011, A $ళ$ A, 528, 124

Dib S., Piau L., Mohanty S., \& Braine J., 2011, MNRAS, 415, 3439

Gardan E., Braine J., Schuster K. F., Brouillet N., \& Sievers A., 2007, A\&\&A, 473, 91

Gratier P., et al., 2012, A\&A 542,108

Gratier P., et al., 2010, A\& $A, 522,3$

Gratier P., Braine J., Rodriguez-Fernandez N. J., Israel F. P., Schuster K. F., Brouillet N., \& Gardan E., 2010, $A \& A, 512,68$ 